\title{
Influência de diferentes agentes de corpo nas características reológicas e sensoriais de chocolates diet em sacarose e light em calorias
}

\author{
Influence of different bulk agents in the rheological and
} sensory characteristics of diet and light chocolate

\author{
Cristiane Rodrigues GOMES ${ }^{1 *}$, Fernanda Zaratini VISSOTTO ${ }^{1}$, \\ Ana Lúcia FADINI ${ }^{1}$, Eliete Vaz de FARIA ${ }^{2}$, Alexandra Motta LUIZ ${ }^{3}$
}

\begin{abstract}
Resumo
Foram estudados diferentes agentes de corpo na substituição total da sacarose na formulação de chocolate, buscando-se obter um produto diet em sacarose e light em calorias (25\% de redução calórica teórica em relação à formulação padrão, com sacarose) e com boa aceitação sensorial. Os agentes de corpo utilizados neste estudo foram: polidextrose, inulina, frutooligossacarídeos - FOS, lactitol e maltitol; como edulcorante de alta intensidade empregou-se a sucralose. Os chocolates light obtidos foram submetidos às análises de umidade (Karl Fisher), tamanho de partículas (micrômetro digital) e propriedades reológicas (viscosidade plástica $-\eta_{\mathrm{ca}}$ e limite de escoamento de Casson - $\tau_{\text {ca }}$ ). $\mathrm{O}$ teor de umidade dos chocolates light variou de 1,23 a 2,12\%; o tamanho de partículas de 19 a $24 \mu \mathrm{m}$; o $\eta_{\text {ca }}$ de 6,60 a 11,00 Pa.s; e o $\tau_{c a}$ de 0,05 a 1,31 Pa. As formulações com polidextrose, polidextrose e lactitol e polidextrose e maltitol foram selecionadas para análise sensorial por apresentarem boa performance tecnológica e adequada maquinabilidade da massa de chocolate durante as diferentes etapas do processo. A análise sensorial indicou que as três formulações avaliadas não diferiram entre si ao nível de significância de $5 \%$ em relação à intensidade do aroma, dureza, derretimento na boca e sabor e, quanto à intenção de compra, as amostras diferiram entre si ao nível de significância de $5 \%$, sendo que a formulação que continha $32,60 \%$ de Polidextrose e $15,57 \%$ de Maltitol foi a preferida.

Palavras-chave: light; diet; chocolate; agentes de corpo; reologia; análise sensorial.
\end{abstract}

\begin{abstract}
A study was made of various bulk agents as sucrose substitutes in the formulation of chocolate, aiming to obtain a diet product in terms of sucrose and a light product in terms of calories ( $25 \%$ fewer calories than standard formulations containing sucrose) with good sensorial acceptance. The bulk agents used in this study were polydextrose, inulin, fructo-oligosaccharides - FOS, lactitol and maltitol. Sucralose was used as a high intensity sweetener. The light chocolates were analyzed for moisture content (Karl Fischer), particle size (digital micrometer), and rheological properties (Casson plastic viscosity, $\eta_{\mathrm{ca}}$, and yield strength, $\tau_{\mathrm{ca}}$ ). The moisture content of the light chocolate varied from 1.23 to $2.12 \%$, while particle size varied from 19 to $24 \mu \mathrm{m}, \eta_{\mathrm{ca}}$ from 6.60 to $11.00 \mathrm{~Pa} . \mathrm{s}$, and $\tau_{\mathrm{ca}}$ from 0.05 to $1.10 \mathrm{~Pa}$. The formulations containing polydextrose, polydextrose and lactitol, and polydextrose and maltitol were selected for a sensory analysis due to their good technological performance and adequate machinability of the chocolate mass in the different stages of the process. The sensory analysis revealed no statistically significant difference $(p>0.05)$ in the three evaluated formulations in terms of aroma, hardness, melting in the mouth and flavor. There was no statistically significant difference $(\mathrm{p}>0.05)$ in the intention to purchase the three chocolate formulations, although a preference was shown for the formulation containing polydextrose (32.60\%) and maltitol (15.57\%).

Keywords: light; diet; chocolate; bulking agents; rheology; sensory analysis.
\end{abstract}

\section{Introdução}

Observa-se, cada vez mais, a tendência crescente do consumo de produtos diet e light no mercado, devido, principalmente, à postura dos consumidores que estão mais conscientes da direta relação da boa alimentação e saúde.

Os chocolates diet atendem ao segmento de diabéticos, hipoglicêmicos e pessoas que desejam ter uma vida mais saudável. Já os chocolates light, se destinam às pessoas propensas à obesidade e às doenças cardiovasculares, causadas por dietas alimentares com altos valores calóricos, ricas em açúcares e gorduras.

Recebido para publicação em 2/10/2006

Aceito para publicação em 18/7/2007 (001871)

Centro de Pesquisa e Desenvolvimento de Chocolates, Balas,

Confeitos e Panificação, CEREAL CHOCOTEC,

Instituto de Tecnologia de Alimentos - ITAL, Av. Brasil, 2880, Jd. Brasil,

CP 139, CEP 13017-178, Campinas - SP, Brasil,

E-mail:cris@ital.sp.gov.br

Laboratório de Análises Físicas e Sensoriais - LAFISE,

Instituto de Tecnologia de Alimentos - ITAL, Av. Brasil, 2880, Jd. Brasil,

CP 139, CEP 13017-178, Campinas - SP, Brasil

Faculdade de Engenharia de Alimentos - FEA,

Universidade Estadual de Campinas - UNICAMP, Campinas - SP, Brasil

*A quem a correspondência deve ser enviada
Estima-se que no final de 2000 havia no mundo cerca de 175,3 milhões de pessoas com diabetes e só na América Latina os diabéticos representavam 16,5 milhões. No Brasil, 7,6\% da população dos 30 aos 69 anos tem diabetes tipo 2, não insulina dependente, o que corresponde à aproximadamente 5 milhões de pessoas ${ }^{8}$.

O balanço calórico positivo tem sido indicado como a principal causa da obesidade em indivíduos saudáveis e consiste na maior ingestão de calorias em relação às calorias gastas. Outras causas da obesidade estão relacionadas com a tendência genética, com as alterações endócrinas e as metabólicas, além dos fatores socioculturais ${ }^{23}$.

Estatísticas do Núcleo de Pesquisas Epidemiológicas em Nutrição e Saúde (Nupens) da Universidade de São Paulo (USP) indicam que nos últimos 20 anos a obesidade entre adultos duplicou e, entre as crianças, triplicou. A grande ameaça da obesidade está na predisposição às doenças cardiovasculares e ao aparecimento do diabetes tipo 2. Dados da Organização Mundial de Saúde (OMS) mostram que no Brasil cerca de $32 \%$ da população está acima do peso e em conseqüência, os 
casos de diabetes tipo 2 têm aumentado drasticamente. Hoje, 17 milhões de brasileiros são portadores da doença e, pelas condições de saúde, outros 40 milhões correm o risco de adquirir a doença nos próximos anos ${ }^{13}$.

Para se obter um chocolate diet e light com qualidade, é necessário o emprego de ingredientes de baixa caloria, capazes de substituir a sacarose, sem causar prejuízo ao sabor e às características físicas quando comparado ao chocolate tradicional. Estes ingredientes substitutos estão divididos em dois grupos distintos de compostos: os edulcorantes de alta intensidade (EAI) e os edulcorantes de baixa intensidade, também denominados de agentes de corpo. O segundo grupo de compostos deve apresentar características similares às da sacarose: reposição de sólidos; estabilidade em diferentes condições de pH e temperatura; ausência de sabor residual; contribuir com a coloração; e interagir com amidos e proteínas de forma similar aos açúcares ${ }^{4}$.

Os agentes de corpo apresentam diferentes características quando comparados entre si. Como exemplos de agentes de corpo existem os polióis (monossacarídeos e dissacarídeos hidrogenados), que possuem dulçor bastante variável e baixa caloria; os substitutos da sacarose poliméricos (a polidextrose e a inulina), que não possuem poder edulcorante; e a frutose, que é um açúcar natural presente nas frutas. Dentre os monossacarídeos hidrogenados destacam-se o sorbitol, o manitol, o xilitol e o eritritol; e entre os dissacarídeos, o isomalte, o maltitol e o lactitol. Todos os açúcares possuem um valor calórico de $4 \mathrm{kcal} . \mathrm{g}^{-1}$. Em contraste, os polióis possuem 2,4 kcal.g ${ }^{-1}$, a polidextrose e a inulina apenas $1 \mathrm{kcal} . \mathrm{g}^{-1}$.

Dentre os critérios para a escolha dos agentes de corpo e edulcorantes de alta intensidade utilizados neste estudo foram considerados o cumprimento de alguns requisitos técnicos (termorresistência, baixa caloria e baixa higroscopicidade), além da disponibilidade no mercado nacional e a aprovação na legislação vigente. Os agentes de corpo relacionados para este estudo foram a polidextrose, a inulina, o FOS, o maltitol e o lactitol, e o edulcorante de alta intensidade, a sucralose.

A polidextrose é um polímero de moléculas de glicose unidas por ligações $\alpha-1,6$, contendo como grupo terminal o sorbitol e/ou ligações monoésteres de ácido cítrico. Para sua produção, são utilizados dextrose, sorbitol e ácido cítrico na proporção 89:10:1, respectivamente ${ }^{5}$.

Dentre os substitutos da sacarose poliméricos, a polidextrose confere corpo, textura e atribui características funcionais semelhantes às da sacarose. Possui alta higroscopicidade, apresenta-se na forma de pó, com coloração creme e não confere sabor e odor aos alimentos, tornando-se necessário seu uso combinado com outros agentes de corpo ou edulcorantes que confiram sabor doce. Uma grande vantagem apresentada pela polidextrose é seu baixo valor calórico se comparado aos carboidratos (polidextrose $=1 \mathrm{kcal} . \mathrm{g}^{-1} \mathrm{e} \mathrm{carboidrato}=4 \mathrm{kcal} . \mathrm{g}^{-1}$ ). Esta baixa caloria é devido à molécula ser grande, complexa e ramificada ao acaso, não sendo possível a sua quebra por enzimas digestivas. Seu metabolismo ocorre apenas por bactérias do intestino, podendo ser, portanto, independentemente da insulina, consumida por diabéticos ${ }^{3,16}$. Em alimentos, observou-se que a polidextrose participa da reação de Maillard, conferindo melhor sabor e escurecimento ao produto, o que é bastante desejável em chocolates, toffees e caramelos ${ }^{19}$.

A polidextrose possui uma atividade de água que se assemelha a da sacarose, sendo, recomendado seu uso na formulação de alimentos que sofreram remoção de ingredientes solúveis como a sacarose, tornando-os microbiologicamente estáveis e com características adequadas de textura. Esse poliol influencia na estabilidade física de doces e produtos de panificação, por atuar na diminuição da taxa de ganho e perda de umidade durante prolongados períodos de armazenamento, sendo, portanto, utilizado visando o aumento da vida-deprateleira dos produtos ${ }^{19}$.

O JECFA estabeleceu "IDA não especificada" para a polidextrose, sendo aprovada em cerca de 60 países. Foi liberada pelo FDA em 1981 para uso em um grande número de produtos, sendo considerado como um carboidrato, porém com valor calórico reduzido $\left(1 \mathrm{kcal} . \mathrm{g}^{-1}\right)$. Deverá constar no rótulo que o consumo excessivo pode causar efeito laxativo em indivíduos sensíveis $^{5}$. No Brasil, a Resolução n ${ }^{0} 386$ de 05 de agosto de 1999, estabelece que o uso da polidextrose está limitado à quantidade necessária para atender às Boas Práticas de Fabricação (BPF), ou seja, quantidade suficiente para obter o efeito tecnológico necessário ${ }^{2}$.

A inulina é uma fibra vegetal que possui um efeito prebiótico no organismo humano, sendo fermentada por bifidobactérias, além de estimular uma maior absorção de cálcio, fatores que a tornam nutricionalmente importante. Trata-se de um carboidrato cuja cadeia é composta predominantemente por unidades de frutose, com uma unidade de glucose terminal $\left(\mathrm{GF}_{\mathrm{n}}\right)$, sendo a ligação entre as moléculas de frutose do tipo $\beta 1,2$, ou seja, uma molécula de sacarose associada a n moléculas de frutose $(\mathrm{n} \approx 30-50)^{5}$.

Uma interessante fonte de inulina é a alcachofra de jerusalém, a qual contém um polifrutano (aproximadamente $75-80 \%$ massa seca) com um grau de polimerização de 3-30 unidades $^{9}$.

A inulina é encontrada em mais de 36.000 plantas. Além da alcachofra, a inulina está presente em quantidades significativas em vegetais como, aspargo, alho-poró, alho e até trigo. Ela também está presente em grandes quantidades nas raízes da chicória ${ }^{18}$.

Ao contrário da maioria dos carboidratos, a inulina não é digerida no estômago. Sua contribuição calórica é pequena: cerca de 1,5 kcal.g ${ }^{-1}$, contra 4 kcal.g ${ }^{-1}$ da sacarose ${ }^{27}$.

Quando ingerida, a inulina chega ao intestino grosso quase que integralmente, não sendo hidrolisada em unidades de monossacarídeos. Como conseqüência, não ocorre aumento da glicemia e nem dos níveis de inulina no sangue, sendo recomendada, portanto, para diabéticos ${ }^{18}$.

A única maneira de a inulina contribuir com o conteúdo calórico é através do metabolismo dos seus produtos de fermentação, realizado no cólon. Contudo, este caminho é menos eficiente do que a absorção dos hidratos de carbono no intestino 
delgado e sua metabolização diretamente no fígado. O consenso hoje é que a inulina tem um valor calórico de $1 \mathrm{kcal} \mathrm{g}^{-1}{ }^{18}$.

A inulina é solúvel em água, mesmo em altas concentrações, não deixando na boca impressão desagradável (arenosa, granulosa). Apresenta boa dispersão nos líquidos, sendo pouco estável a pH inferior a 4 e a altas temperaturas, substituindo matérias graxas sem grandes modificações no processo de fabricação, podendo ser usada como substituto de açúcar em chocolates. A combinação da inulina com os polióis e com a frutose apresenta excelentes resultados ${ }^{1}$.

Comercialmente a inulina é vendida com o nome de Raftiline ${ }^{\circledast}$, sendo considerada como um alimento e não um aditivo. Doses inferiores a 20 g/refeição não causam efeitos colaterais, superiores a $60 \mathrm{~g} /$ dia podem apresentar efeito laxativo ${ }^{5}$.

Frutooligossacarídeos (FOS) são oligômeros da 1-(1$\beta$-frutofuranosil) ${ }_{\mathrm{n}-1}$ sacarose, nos quais n varia de 2 a 4 . Consistem em moléculas de sacarose às quais adicionam-se uma, duas ou três unidades de frutose através de ligação glicosídica $\beta$ $(2,1)$ na unidade de frutose da sacarose, reduzindo ao mínimo o teor de glicose ${ }^{5}$.

Raftilose $^{\circledR}$ é o nome comercial de xaropes e pós contendo oligofrutose (frutooligossacarídeos), obtidos da hidrólise enzimática da inulina. Apresentam propriedades tecnológicas semelhantes aos xaropes de glicose e frutose. Seu sabor é neutro e moderadamente doce. O valor calórico é de aproximadamente $1,5 \mathrm{kcal} \cdot \mathrm{g}^{-1}$.

O maltitol não ocorre na natureza. É produzido industrialmente baseado na hidrogenação catalítica da maltose, produzida pela hidrólise do amido ${ }^{12}$.

O poder edulcorante deste poliol (na forma cristalina com $98 \%$ de pureza) é de aproximadamente 80 a $90 \%$ o da sacarose. A habilidade de conferir "corpo", a solubilidade, o calor de dissolução, os efeitos de atividade de água e de depressão do ponto de congelamento são semelhantes aos da sacarose. O maltitol possui alta higroscopicidade, exigindo cuidados especiais quanto à embalagem. Tem boa estabilidade química, térmica e enzimática e não deixa sensação refrescante ou sabor residual ${ }^{5}$.

Em seres humanos, o metabolismo do maltitol fornece 50\% das calorias dos carboidratos, sendo estas calorias obtidas, principalmente, pela fermentação do maltitol por bactérias da microflora intestinal produzindo ácidos graxos voláteis, que serão absorvidos e metabolizados ${ }^{17}$. Pela legislação brasileira, o maltitol contribui com 2,4 kcal.g ${ }^{-1}$.

Ao maltitol foi conferida, pelo JECFA, "IDA não específicada”, sendo que a ingestão de até 50 g/dia não causa transtornos intestinais ${ }^{5}$.

O lactitol descoberto em 1920 e sintetizado com êxito em 1937, é um poliol dissacarídeo, o 4-O- $\beta$-D-galactopiranosilD-sorbitol $\left(\mathrm{C}_{12} \mathrm{H}_{24} \mathrm{O}_{11} \cdot \mathrm{H}_{2} \mathrm{O}\right)$. É obtido através da hidrogenação catalítica de uma solução de lactose (açúcar do leite) a 30-40\% por redução de uma unidade glicopiranosil em sorbitol ${ }^{5}$.

O lactitol é comumente usado como agente de corpo em alimentos com controle de calorias ${ }^{11}$. Devido à sua baixa doçura
(0,3 - 0,4 vezes da sacarose) é industrialmente utilizado como suporte e estabilizador de aromas e em produtos farmacêuticos. Mostra perfil de doçura semelhante ao da sacarose com dulçor suave e sem sabor residual ${ }^{5}$. Sua doçura intermediária faz necessária a combinação com edulcorantes de alta intensidade (e baixa caloria) como o aspartame, a sacarina e o acessulfame-k.

O lactitol não é metabolizado pelas bactérias da boca, logo não causa cáries. Ao contrário do metabolismo da lactose, o lactitol não é hidrolisado pela lactase. Também não é hidrolisado ou absorvido no intestino delgado. O lactitol é metabolizado por bactérias e convertido em biomassa, ácidos orgânicos, dióxido de carbono e uma pequena concentração de hidrogênio ${ }^{11}$. Como um ingrediente adoçante, o lactitol não induz ao aumento da glicose sanguínea ou insulina, e contribui com apenas metade das calorias dos carboidratos. Pela legislação brasileira, o lactitol contribui com apenas 2,4 kcal.g ${ }^{-1}{ }^{11}$.

O lactitol forma cristais sem cor ou odor e não é higroscópico, ou seja, não absorve umidade, mantendo o frescor e aumentando o tempo de vida de biscoitos e gomas de mascar (a forma anidra é higroscópica e absorve mais água que o xilitol). É um pouco menos solúvel que o sorbitol e mais estável que a lactose. Não tem um grupo carboxil livre, não sofre reação de Maillard e se decompõe apenas a temperaturas acima de $200{ }^{\circ} \mathrm{C}$.

O lactitol é prontamente solúvel em água e miscível com outros polióis e carboidratos, sendo suas soluções levemente menos viscosas que as de lactose em mesma concentração ${ }^{10}$.

O Comitê Científico para Alimentos da UE e o JECFA estabeleceram "IDA não especificada" para o lactitol, porém para evitar efeito laxativo não deve ser excedida a dose de $20 \mathrm{~g} /$ dia. $\mathrm{O}$ organismo é capaz de se acostumar com o lactitol num período de tempo relativamente curto, permitindo o consumo de doses superiores à recomendada ${ }^{5}$.

A sucralose foi desenvolvida em 1976, por pesquisadores da Tate Lyle Specialty Sweeteners (Inglaterra) e pelo grupo do Professor Hough da Queen Elizabeth College, na Universidade de Londres ${ }^{5}$.

A sucralose é o único adoçante não calórico produzido a partir do açúcar, não sendo percebido nenhum sabor residual; podendo ser usado em praticamente todos os produtos nos quais usa-se açúcar, incluindo produtos assados e cozidos ${ }^{21}$. Seu poder adoçante é cerca de 600 vezes o da sacarose, não é metabolizado pelo organismo (portanto pode ser ingerido por diabéticos) e é estável a largas faixas de temperatura e $\mathrm{pH}^{22}$.

Na determinação da inocuidade da sucralose, o FDA analisou mais de 110 estudos em seres humanos e animais. A maioria dos estudos estava voltado para identificar possíveis efeitos tóxicos, incluindo carcinogênicos, reprodutivos e neurológicos. Nenhum efeito colateral foi encontrado, e a aprovação da FDA está baseada na seguridade da sucralose para os seres humanos ${ }^{7}$.

A ingestão diária admissível (IDA) para a sucralose foi fixada em $15 \mathrm{mg}$ por quilograma de peso corpóreo $^{26}$. 
O objetivo deste trabalho de pesquisa foi o de estudar diferentes agentes de corpo na substituição total da sacarose na formulação de chocolate, buscando-se obter um produto diet em sacarose e light em calorias (25\% de redução calórica teórica em relação à formulação padrão, com sacarose) e com boa aceitação sensorial.

\section{Material e métodos}

\subsection{Material}

Como o objetivo do presente trabalho foi produzir um chocolate que além de diet em sacarose fosse light em calorias, o seu valor calórico deveria, necessariamente, ser $25 \%$ menor que uma média calórica de três produtos comerciais. Para tanto, fez-se uma pesquisa de mercado, sendo a média do valor calórico de três marcas de chocolate ao leite em tablete considerada o padrão para a redução.

Os chocolates dietéticos foram formulados utilizando-se a manteiga de cacau $(21,90 \%)$, o líquor de cacau $(11,20 \%)$, o leite em pó integral (11,00\%) e o desnatado $(7,00 \%)$, a lecitina de soja (0,30\%), o Poliglicerol Polirricinoleato - PGPR (0,20\%), o aroma vanila $(0,10 \%)$, a sucralose $(0,03 \%)$ e os agentes de corpo $(48,27 \%)$. Os agentes de corpo utilizados nos experimentos e suas combinações são apresentados na Tabela 1. Foi também formulado um chocolate regular (formulação padrão) com o intuito de compará-lo aos dietéticos no que diz respeito ao valor calórico.

\subsection{Métodos}

Foi realizada a mistura do líquor de cacau com o leite em pó (desnatado e integral), 2/3 da manteiga de cacau da formulação e os agentes de corpo e o edulcorante de alta intensidade, no caso de chocolate dietético; a sacarose foi utilizada na formulação do chocolate padrão. Empregou-se nesta etapa um misturador planetário Inco, e o processo foi realizado à temperatura ambiente, por 15 minutos.

O refino foi feito em moinho piloto de três cilindros Draiswerk GMBH e o tamanho de partículas medido em micrômetro digital Mitutoyo.

A conchagem foi realizada em concha longitudinal Friwessa, sendo a temperatura de conchagem de $55{ }^{\circ} \mathrm{C}$ mantida constante por 17 horas. O 1/3 restante da manteiga de cacau foi adicionado no início desta etapa, juntamente com os emulsificantes, sendo o aroma de baunilha adicionado ao final do processo. Depois da conchagem a umidade do chocolate foi medida empregando-se o método oficial A.O.A.C. 31.1.0320. Aproximadamente 0,5 g de chocolate (pesado em balança analítica) foi dissolvido em uma solução de clorofórmio:metanol, na proporção 1:1. O equipamento para a determinação da umidade foi o Karl Fischer (Titroline Alpha/Schott-Gerate GmbH TM 125), sendo os resultados apresentados neste trabalho a média de três determinações.

\section{Propriedades reológicas}

As amostras acondicionadas em recipientes de vidro com tampa foram fundidas em estufa a $45^{\circ} \mathrm{C}$. Para a determinação das propriedades reológicas, utilizou-se um reômetro programável, Brookfield, modelo RVDVIII, com adaptador de pequenas amostras e spindle do tipo cilíndrico (especificação: S15). Um banho termostático Brookfield foi acoplado ao adaptador de pequenas amostras com o objetivo de manter a temperatura do produto constante e igual a $40{ }^{\circ} \mathrm{C}$. As rotações do spindle foram estabelecidas segundo VISSOTTO et al. ${ }^{24}$.

Segundo CHEVALLEY ${ }^{6}$, a utilização de um reômetro rotativo de cilindros coaxiais faz necessária à inclusão da relação entre os raios dos cilindros interno e externo no modelo de Casson:

$(1+\mathrm{a}) \sqrt{\mathrm{D}_{\mathrm{n}}}=\frac{1}{\sqrt{\eta_{\mathrm{ca}}}}\left[(1+\mathrm{a}) \sqrt{\tau}-2 \sqrt{\tau_{\mathrm{ca}}}\right]$

onde:

$\mathrm{D}_{\mathrm{n}}=$ taxa de deformação aparente $\left(\mathrm{s}^{-1}\right)$;

$a$ = quociente dos raios dos cilindros interno e externo;

$\tau=$ tensão de cisalhamento na face externa do cilindro interno $(\mathrm{Pa})$;

$\tau_{\text {ca }}=$ limite de escoamento de Casson (Pa); e

$\eta_{\mathrm{ca}}=$ viscosidade plástica de Casson (Pa.s).

Os dados obtidos do reômetro são graficados de forma que o termo $(1+$ a $) \sqrt{D n}$ fique na ordenada e o termo $(1+$ a $) \sqrt{ } \tau$ fique na abscissa. Aos pontos experimentais ajusta-se, por regressão linear, uma reta com inclinação igual a $1 / \eta$ CA e intersecção com a abscissa igual a $2 \sqrt{ } \tau \mathrm{CA}$.

\section{Análise sensorial}

As formulações com polidextrose, polidextrose e lactitol e polidextrose e maltitol foram selecionadas para análise sensorial por apresentarem boa performance tecnológica e adequada maquinabilidade da massa de chocolate durante as diferentes

Tabela 1. Proporção dos agentes de corpo nas formulações.

\begin{tabular}{|c|c|c|c|c|c|c|c|c|c|}
\hline \multirow[t]{2}{*}{ Agentes de corpo (\%) } & \multicolumn{9}{|c|}{ Formulações } \\
\hline & Padrão & 1 & 2 & 3 & 4 & 5 & 6 & 7 & 8 \\
\hline Sacarose & 48,27 & - & - & - & - & - & - & - & - \\
\hline Polidextrose & - & 48,27 & 32,60 & 26,27 & 24,14 & - & - & - & 32,60 \\
\hline Lactitol & - & - & 15,57 & 12,00 & - & - & 15,57 & - & - \\
\hline Maltitol & - & - & - & - & - & - & - & 15,57 & 15,57 \\
\hline Inulina & - & - & - & - & 24,13 & 48,27 & 32,60 & 32,60 & - \\
\hline FOS & - & - & - & 10,00 & - & - & - & - & - \\
\hline
\end{tabular}


etapas do processo. As amostras foram avaliadas por meio de teste afetivo quanto à intensidade dos atributos aroma, dureza, derretimento na boca e sabor por meio de escalas de intensidade de sete pontos $(7=$ muito forte, macio ou rápido, $4=$ nem forte nem fraco, nem macio nem duro ou nem rápido nem demorado e 1 = muito fraco, muito duro ou muito demorado, de acordo com os atributos considerados), solicitando-se a descrição de gostos e desgostos associados a cada amostra. A aceitabilidade das amostras foi avaliada por meio de escala de intenção de compra de cinco pontos ( 5 = certamente compraria, 3 = não sei e 1 = certamente não compraria $)^{14}$. O modelo de ficha de avaliação utilizado é apresentado na Figura 1.

As amostras foram avaliadas de forma monádica seqüencial segundo um delineamento de blocos completos casualizados, sendo apresentadas com códigos de três números aleatórios. O teste foi conduzido em cabines individuais com iluminação de lâmpadas fluorescentes e equipadas com o siste-

Avaliação de chocolate diet e light ao leite

Nome: Produto: 285

Você receberá três amostras de chocolate ao leite light e diet, uma de cada vez. Por favor prove as amostras e responda as questões a seguir:

1. Indique como você classifica o produto em relação aos seguintes aspectos:

Aroma

Muito Moderadamente Ligeiramente Nem forte Ligeiramente Moderadamente Muito forte forte forte nem fraco fraco r fraco fraco

Dureza

Muito Moderadamente Ligeiramente Nem macio Ligeiramente Moderadamente Muito macio macio macio nem duro duro $\quad$ duro duro

Derretimento na boca

Muito Moderadamente Ligeiramente Nem rápido Ligeiramente Moderadamente Muito rápido rápido rápido nem demorado demorado demorado demorado

Sabor

Muito Moderadamente Ligeiramente Nem forte Ligeiramente Moderadamente Muito forte forte forte nem fraco fraco fraco fraco

2. Por favor, descreva o que em particular você gostou ou desgostou nesta amostra.

Mais gostei:

Menos gostei:
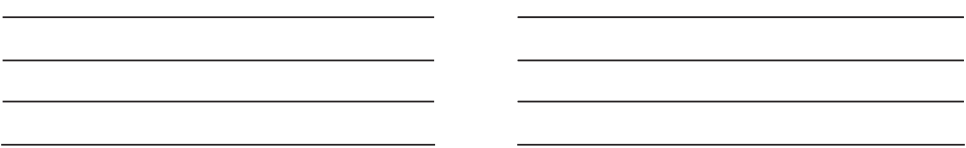

3. Indique qual seria sua atitude em relação à compra do produto que acabou de provar:

\begin{tabular}{|c|c|c|c|c|}
\hline $\begin{array}{r}\text { Certamente } \\
\text { compraria }\end{array}$ & $\begin{array}{l}\text { Provavelmente } \\
\text { compraria }\end{array}$ & Não sei & $\begin{array}{l}\text { Provavelmente } \\
\text { não compraria }\end{array}$ & $\begin{array}{c}\text { Certamente não } \\
\text { compraria }\end{array}$ \\
\hline
\end{tabular}

Figura 1. Ficha de avaliação utilizada no teste afetivo. 
ma computadorizado Compusense Five versão 4.6 para coleta e análise dos dados. Os dados relativos às escalas utilizadas foram submetidos à análise de variância e teste de Tukey para comparação de médias, além de ser calculada a porcentagem de respostas associadas às categorias acima do ponto médio, ao ponto médio e abaixo do ponto médio das escalas utilizadas. Os comentários descritos como gostos e desgostos em relação a cada amostra foram agrupados visando estabelecer a freqüência de respostas acumuladas como percepções similares por parte dos consumidores consultados. Para o teste afetivo, foram recrutados 30 consumidores de chocolate e de produtos light e/ou diet em geral, com faixa etária de 18 a 35 anos.

\section{Resultados e discussão}

\subsection{Determinação teórica dos valores calóricos}

O valor calórico dos tabletes das marcas consideradas foi:

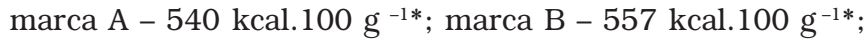
marca C - $560 \mathrm{kcal} .100 \mathrm{~g}^{-1 *}$; e o valor calórico médio dos produtos, $552,33 \mathrm{kcal} 100 \mathrm{~g}^{-1}$. O chocolate a ser desenvolvido deveria conter, portanto, no máximo 414,25 kcal.100 g ${ }^{-1}$.

Na determinação dos valores calóricos das formulações foi realizado um levantamento com referência aos ingredientes e aditivos, utilizando-se as tabelas nutricionais fornecidas pelas empresas (Tabela 2).

Tabela 2. Valor calórico dos ingredientes usados nas formulações.

\begin{tabular}{lc}
\hline \multicolumn{1}{c}{ Ingrediente } & Valor calórico $\left(\mathrm{kcal} .100 \mathrm{~g}^{-1}\right)$ \\
\hline Leite em pó desnatado & 357,0 \\
Leite em pó integral & 490,0 \\
Lecitina de soja & 875,0 \\
Aroma de baunilha & $\cong 0,0$ \\
Manteiga de cacau & 900,0 \\
Líquor de cacau natural & 567,2 \\
PGPR & 850,0 \\
Inulina & 100,0 \\
Polidextrose & 100,0 \\
Lactitol/maltitol & 240,0 \\
FOS & 150,0 \\
Sucralose & $\cong 0,0$ \\
\hline
\end{tabular}

Na Tabela 3, são apresentados os valores calóricos e a redução calórica das 8 formulações desenvolvidas.

Tabela 3. Valores calóricos e a redução calórica das formulações.

\begin{tabular}{ccc}
\hline Formulação & $\begin{array}{c}\text { Valor calórico } \\
\left(\text { kcal. } 100 \mathrm{~g}^{-1}\right)\end{array}$ & $\begin{array}{c}\text { Redução calórica } \\
(\%)\end{array}$ \\
\hline 1 & 392,11 & 29,01 \\
2 & 413,91 & 25,06 \\
3 & 413,91 & 25,06 \\
4 & 392,11 & 29,01 \\
5 & 392,11 & 29,01 \\
6 & 413,91 & 25,06 \\
7 & 413,91 & 25,06 \\
8 & 413,91 & 25,06 \\
\hline
\end{tabular}

Portanto, todas as formulações apresentadas neste trabalho de pesquisa atendem ao requisito de serem diet em sacarose e light em calorias.

\subsection{Caracterização física e físico-química das amostras}

O tamanho de partícula das amostras foi medido depois da conchagem, considerando-se que o equipamento utilizado no processo, a concha longitudinal, pode ocasionar uma pequena diminuição no tamanho das partículas da massa de chocolate. Os valores dos tamanhos das partículas variaram entre 19,0 e 24,0 $\mu \mathrm{m}$, estando dentro de uma faixa adequada para chocolates, visto que valores superiores a $25 \mu \mathrm{m}$ são perceptíveis ao paladar.

Também, após a conchagem, fez-se a determinação da umidade da massa de chocolate utilizando-se método de análise com reagente Karl Fisher, bastante indicado para produtos que apresentam teor baixo de umidade, como é o caso dos chocolates. Os resultados obtidos oscilaram entre 1,29 e $2,12 \%$. Segundo MINIFIE ${ }^{15}$, o chocolate ao leite deve apresentar um teor de umidade entre 0,50 e $1,50 \%$. Valores superiores podem ocasionar aumento da viscosidade do produto. Durante o estudo observou-se que algumas amostras apresentaram umidade acima da recomendada (formulações 1, 4, 5, 6 e 7), possivelmente devido à presença de ingredientes higroscópicos (principalmente a polidextrose e a inulina) que poderiam dificultar a eliminação da umidade durante a etapa de conchagem e favorecer a sua absorção durante o período de estocagem.

\subsection{Propriedades reológicas}

Os parâmetros reológicos viscosidade plástica de Casson $\eta_{\text {ca }}$ e limite de escoamento de Casson - $\tau_{\text {ca }}$, das formulações estudadas, assim como os coeficientes de correlação linear $\left(\mathrm{R}^{2}\right)$ são apresentados na Tabela 4. Os coeficientes de correlação demonstram bom ajuste dos dados experimentais ao modelo de Casson, visto que apresentaram variação apenas na quarta casa decimal.

Tabela 4. Caracterização reológica das formulações light e diet testadas.

\begin{tabular}{cccc}
\hline Amostra & $\begin{array}{c}\text { Viscosidade plástica de } \\
\text { Casson } \eta_{\mathrm{ca}}(\mathrm{Pa} . \mathrm{s})^{*}\end{array}$ & $\begin{array}{c}\text { Limite de escoamento } \\
\text { de Casson } \tau_{\mathrm{ca}}(\mathrm{Pa})^{*}\end{array}$ & $\mathrm{R}^{2}$ \\
\hline 1 & $11,04 \pm 0,72$ & $0,17 \pm 0,01$ & 0,9991 \\
2 & $6,64 \pm 0,31$ & $0,08 \pm 0,01$ & 0,9993 \\
3 & $8,20 \pm 0,20$ & $0,41 \pm 0,05$ & 0,9993 \\
4 & $8,22 \pm 0,14$ & $1,31 \pm 0,09$ & 0,9996 \\
5 & $7,55 \pm 0,13$ & $0,58 \pm 0,02$ & 0,9996 \\
6 & $7,57 \pm 0,34$ & $0,05 \pm 0,00$ & 0,9996 \\
7 & $7,59 \pm 0,39$ & $0,17 \pm 0,01$ & 0,9997 \\
8 & $9,67 \pm 0,27$ & $0,09 \pm 0,01$ & 0,9997 \\
\hline${ }^{*}$ Média \pm desvio padrão de três repetições. & &
\end{tabular}

Analisando-se a Tabela 4, observa-se que as formulações de chocolate light e diet possuem $\eta_{\mathrm{ca}}$ entre 6,64 e 11,04 Pa.s e valores de $\tau_{\text {ca }}$ entre 0,05 e 1,31 Pa. Os baixos valores de $\tau_{\text {ca }}$ obtidos para quase todas as amostras devem-se principalmente ao uso do Poliglicerol Polirricinoleato - PGPR, agente emulsificante que age diminuindo o limite de escoamento dos produtos.

Os valores encontrados neste estudo estão bastante próximos dos determinados por VISSOTTO, GOMES e BATISTA ${ }^{25}$ 
para chocolates diet $\left(\eta_{\text {ca }}\right.$ entre 4,48 e 17,08 Pa.s e $\tau_{\text {ca }}$ entre 0,01 e 2,00 Pa).

Não foram encontrados na literatura parâmetros reológicos para chocolates light em calorias.

\subsection{Análise sensorial}

Os resultados médios obtidos no teste afetivo para avaliação da intensidade dos atributos aroma, dureza, derretimento na boca e sabor e da intenção de compra das Formulações 1, 2 e 8 são apresentados na Tabela 5. Verifica-se que estas três formulações não diferiram entre si ao nível de 5\% de significância em relação à intensidade do aroma, dureza, derretimento na boca e sabor, para os quais os valores médios obtidos correspondem aos pontos médios das escalas utilizadas, isto é, aroma e sabor "nem forte nem fraco", dureza "nem macio nem duro", derretimento na boca "nem rápido nem demorado". Quanto à intenção de compra, as amostras diferiram entre si ao nível de 5\% de significância, sendo que a Formulação 8 obteve média correspondente à "provavelmente compraria" na escala utilizada, enquanto para a Formulação 2 o valor médio situa-se entre "provavelmente compraria" e "não sei" e a Formulação 1 obteve média correspondente a "não sei".

Nas Figuras 2 e 3, são apresentadas as distribuições em freqüência dos valores da escala atribuídos pelos consumidores aos produtos e, na Tabela 6, são apresentadas as porcentagens de respostas associadas às categorias acima do ponto médio, ao ponto médio e abaixo do ponto médio das escalas utilizadas, correspondendo à classificação como forte, nem fraco nem forte e fraco para o aroma e sabor, à macio, nem macio nem duro e duro para a dureza, à rápido, nem rápido nem demorado e demorado para o derretimento na boca e à intenção de compra positiva, duvidosa ou negativa. As freqüências de menções positivas e negativas descritas pelos consumidores ao relatarem o que mais gostaram e desgostaram nas amostras são apresentadas na Figura 4.

A análise das informações contidas nas Figuras 2 a 4 e na Tabela 6 permitem diagnosticar a melhor intenção de compra associada à Formulação 8, já que as escalas de intensidade não forneceram informações sobre as percepções dos consumidores quanto às diferenças sensoriais entre as amostras. Com relação à intensidade do aroma, aproximadamente 50\% dos consumidores consultados consideraram as três amostras com aroma "nem fraco nem forte"; com relação à dureza, a Formulação 1 foi considerada "nem macia nem dura" por 53,3\% dos consumidores, enquanto as Formulações 2 e 8 foram consideradas com textura dura por boa parte dos consumidores (40,0 a 46,6\%).

A Formulação 1 foi considerada com derretimento na boca demorado por mais de $50 \%$ dos consumidores, sendo que as Formulações 2 e 8 obtiveram esta classificação por 36,7 e 40,0\% dos consumidores, respectivamente.

A Formulação 8 foi classificada com sabor "nem forte nem fraco" por $50 \%$ dos consumidores, sendo que somente $13,3 \%$

Tabela 5. Resultados obtidos no teste afetivo para avaliação da intensidade dos atributos aroma, dureza, derretimento na boca e sabor e indicação da intenção de compra por 30 consumidores de chocolate e produtos light/diet em geral. Em cada coluna, valores seguidos de letras iguais não diferem estatisticamente entre si ao nível de erro de $5 \%$ *

\begin{tabular}{|c|c|c|c|c|c|}
\hline & \multicolumn{4}{|c|}{ Intensidade } & \multirow[t]{2}{*}{ Intenção de compra } \\
\hline & Aroma & Dureza & Derretimento na boca & Sabor & \\
\hline Formulação 1 & $(3,8 \pm 1,2)^{\mathrm{a}}$ & $(4,0 \pm 1,0)^{\mathrm{a}}$ & $(3,3 \pm 1,2)^{\mathrm{a}}$ & $(4,0 \pm 1,6)^{a}$ & $(2,8 \pm 1,2)^{\mathrm{c}}$ \\
\hline Formulação 2 & $(4,1 \pm 1,1)^{\mathrm{a}}$ & $(4,0 \pm 1,2)^{\mathrm{a}}$ & $(3,8 \pm 1,3)^{\mathrm{a}}$ & $(4,1 \pm 1,3)^{\mathrm{a}}$ & $(3,5 \pm 1,2)^{\mathrm{b}}$ \\
\hline Formulação 8 & $(4,1 \pm 1,2)^{\mathrm{a}}$ & $(3,8 \pm 1,2)^{\mathrm{a}}$ & $(3,8 \pm 1,1)^{\mathrm{a}}$ & $(4,2 \pm 0,8)^{\mathrm{a}}$ & $(4,1 \pm 0,8)^{\mathrm{a}}$ \\
\hline D.M.S. & 0,58 & 0,65 & 0,59 & 0,65 & 0,53 \\
\hline
\end{tabular}

*Resultado expresso como média \pm desvio padrão; e D.M.S.: diferença mínima significativa ao nível de erro de $5 \%$ pelo Teste de Tukey.

Tabela 6. Porcentagens associadas às categorias acima do ponto médio, ao ponto médio e abaixo do ponto médio das escalas utilizadas.

\begin{tabular}{|c|c|c|c|c|}
\hline \multirow[t]{2}{*}{ Atributos } & \multirow[t]{2}{*}{ Classificação } & \multicolumn{3}{|c|}{ Amostras } \\
\hline & & Formulação 1 & Formulação 2 & Formulação 8 \\
\hline \multirow[t]{3}{*}{ Intensidade do aroma } & Forte $(\%)$ & 26,7 & 26,6 & 30,0 \\
\hline & Nem fraco nem forte (\%) & 46,7 & 46,7 & 50,0 \\
\hline & Fraco (\%) & 26,6 & 26,7 & 20,0 \\
\hline \multirow[t]{3}{*}{ Dureza } & Macio (\%) & 23,4 & 26,6 & 33,4 \\
\hline & Nem macio nem duro (\%) & 53,3 & 33,4 & 20,0 \\
\hline & Duro (\%) & 23,3 & 40,0 & 46,6 \\
\hline \multirow[t]{3}{*}{ Derretimento na boca } & Rápido (\%) & 20,0 & 30,0 & 26,7 \\
\hline & Nem rápido nem demorado (\%) & 23,3 & 33,3 & 33,3 \\
\hline & Demorado (\%) & 56,7 & 36,7 & 40,0 \\
\hline \multirow[t]{3}{*}{ Intensidade do sabor } & Forte $(\%)$ & 40,0 & 40,0 & 36,6 \\
\hline & Nem fraco nem forte (\%) & 26,7 & 26,7 & 50,0 \\
\hline & Fraco (\%) & 33,3 & 33,3 & 13,3 \\
\hline \multirow[t]{3}{*}{ Intenção de compra } & Positiva (\%) & 26,7 & 56,7 & 86,7 \\
\hline & Duvidosa (\%) & 30,0 & 20,0 & 6,7 \\
\hline & Negativa (\%) & 43,4 & 23,4 & 6,7 \\
\hline
\end{tabular}



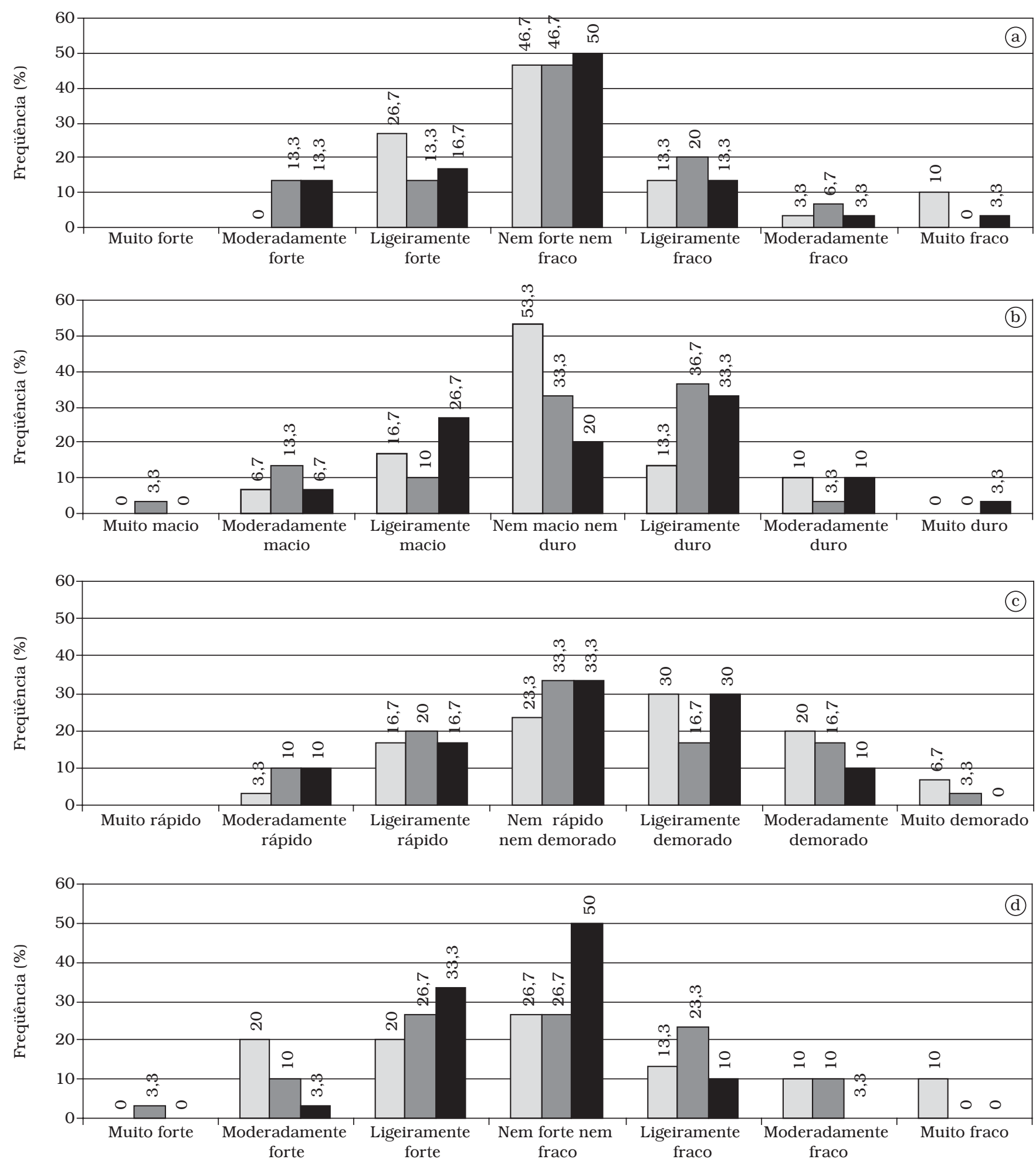

Formulação $1 \square$ Formulação $2 \square$ Formulação 8

Figura 2. Distribuição em freqüência dos valores da escala atribuídos pelos consumidores às amostras de chocolate diet/light quanto à intensidade dos atributos a) aroma; b) dureza; c) derretimento na boca; e d) sabor.

consideraram fraco o seu sabor em contraposição à classificação do sabor das Formulações 1 e 2, para as quais 33,3\% dos consumidores consideram o sabor fraco.
A Formulação 8 recebeu maior número de menções positivas e menor número de menções negativas do que as Formulações 1 e 2 (Figura 4), sendo que o sabor e a textura 


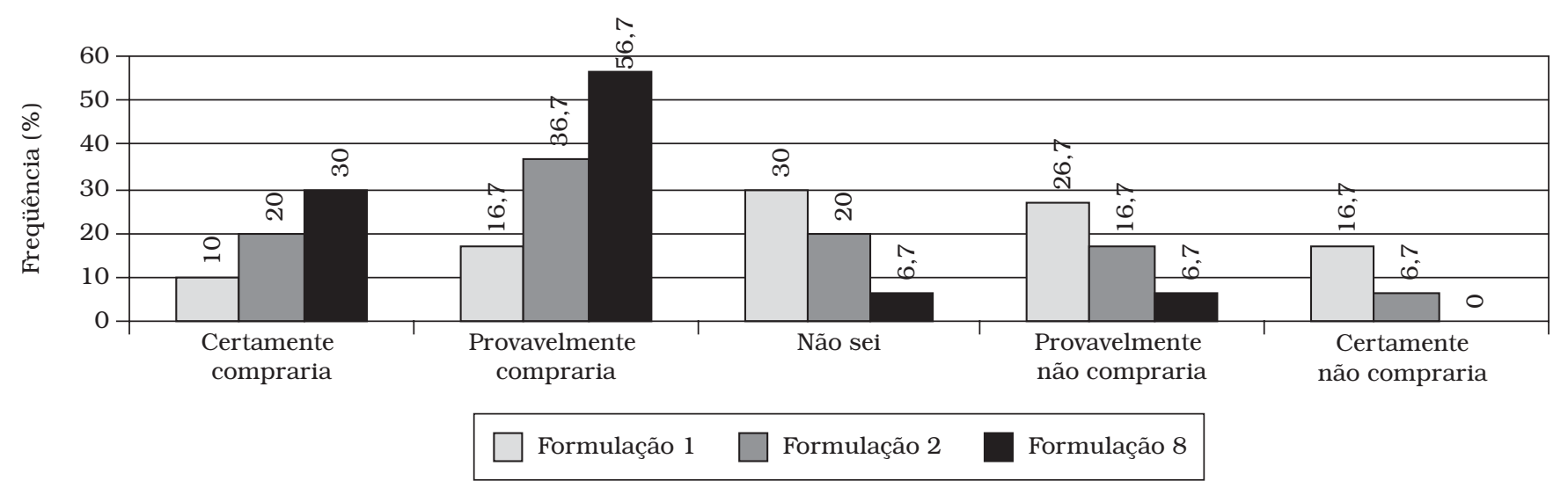

Figura 3. Distribuição em freqüência dos valores da escala atribuídos pelos consumidores às amostras de chocolate diet/light quanto à intenção de compra.

(a)

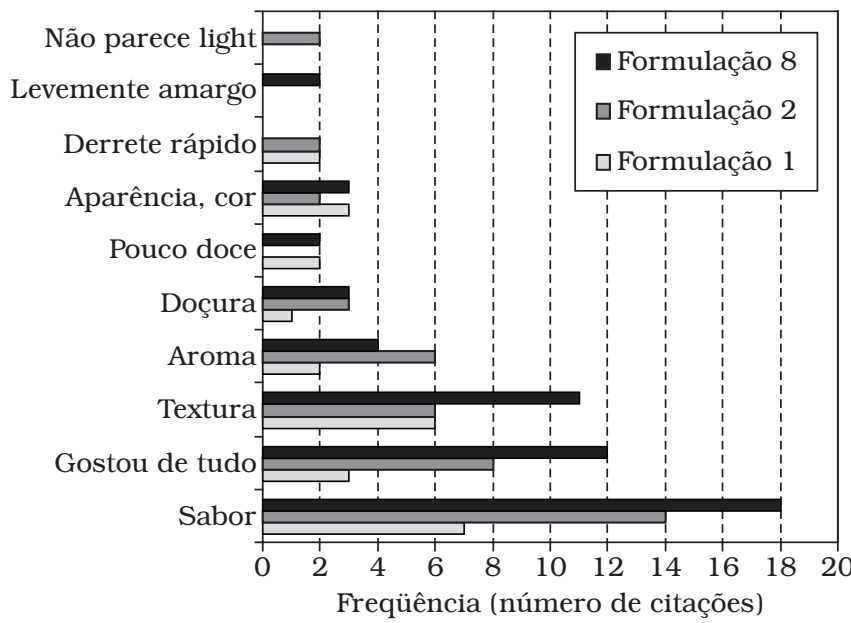

(b)

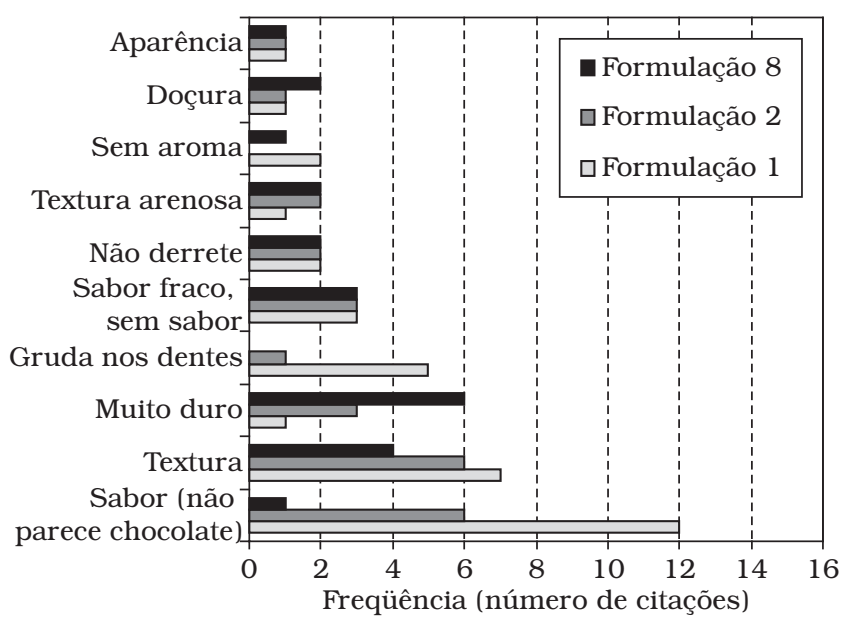

Figura 4. Distribuição em freqüência dos comentários relativos à questão aberta a) "mais gostei" e b) "menos gostei" descritos pelo grupo de 30 consumidores para as amostras de chocolate diet/light. destacaram-se como ponto forte da Formulação 8, além de $40 \%$ dos consumidores consultados terem relatado que gostaram de tudo nesta amostra.

Em geral, o ponto fraco da Formulação 1, traduzido pela maior freqüência de menções negativas, é o sabor, descrito como não parecer ser de chocolate por $40 \%$ dos consumidores consultados, enquanto para a Formulação 2, o sabor e a textura foram igualmente apontados negativamente, com indicação, por $16,7 \%$ dos consumidores, de que a Formulação 1 gruda nos dentes. A Formulação 8 foi considerada como muito dura por $20 \%$ dos consumidores consultados.

Essas observações permitem concluir que o sabor da Formulação 8, menos fraco do que o das demais amostras, associado à ausência do conjunto de aspectos negativos percebidos nas Formulações 1 e 2, explica a intenção de compra superior atribuída à Formulação 8 em relação às demais.

\section{Conclusões}

O estudo atingiu seus objetivos quanto à obtenção de chocolates isentos de sacarose (diet) e com $25 \%$ de redução do valor calórico (light).

Algumas amostras apresentaram umidade acima da recomendada para chocolate (formulações 1, 4, 5, 6 e 7), possivelmente devido à presença de ingredientes higroscópicos (principalmente a polidextrose e a inulina) que poderiam dificultar a eliminação da umidade durante a etapa de conchagem e favorecer a sua absorção durante o período de estocagem.

Os parâmetros reológicos dos chocolates desenvolvidos mostraram bom ajuste dos dados experimentais ao modelo de Casson, podendo ser representados por meio dos parâmetros limite de escoamento de Casson $\left(\eta_{\mathrm{ca}}\right)$ e viscosidade plástica de Casson $\left(\tau_{\text {ca }}\right)$.

Embora as Formulações 1, 2 e 8 não tenham diferido entre si ao nível de $5 \%$ de significância em relação à intensidade do aroma, dureza, derretimento na boca e sabor, a Formulação 8 obteve intenção de compra superior às demais ao nível de $5 \%$ de significância, com indicação de que os consumidores consultados consideraram seu sabor menos fraco do que o das 
demais amostras, o que, associado à ausência do conjunto de aspectos negativos percebidos nas Formulações 1 e 2, tais como sabor que não parece ser de chocolate e grudar nos dentes, explica sua melhor aceitação.

\section{Agradecimento}

Ao Conselho Nacional de Pesquisa e Desenvolvimento Científico (CNPq) pelo apoio financeiro para execução do projeto através da concessão de bolsa de iniciação científica.

\section{Referências bibliográficas}

1. A INULINA, uma grande desconhecida. Aditivos \& Ingredientes, n. 8, p. 36-41, maio/junho, 2000.

2. BRASIL. Resolução ${ }^{\circ} 386$ de 05 agosto de 1999. Aprova o regulamento técnico sobre "Aditivos utilizados segundo as boas práticas de fabricação e suas funções". Disponível em: $<$ http:// www.anvisa.gov.br/legis/resol/386_99.htm > Acesso em: 11 jan. 2006.

3. BUNTING, C. SugarFree Ingredient. The Manufacturing Confectioner, Outubro, 1994, p. 55-58.

4. CAMPOS, M. B. Chocolates sem adição de açúcar: matériasprimas, formulações, processo de produção e análise sensorial. In: Manual Técnico do Seminário Produtos Diet e Light, Campinas, Junho de 2000, 154 p.

5. CÂNDIDO, L. M. N.; CAMPOS, A. M. Alimentos para fins especiais: diabéticos. São Paulo: Livraria Varela, 1996. 423 p.

6. CHEVALLEY, J. Rheology of chocolate. Journal of texture studies, v. 6, n. 1, p. 176-196, 1974.

7. FDA APPROVES. Disponível em <http://vm.cfsan.fda.gov $\sim$ Ird $>$ Acesso em: 02 agost. 2006.

8. GARCIA, A. E. B. Tendências de mercado para produtos diet e light no setor de chocolates, balas e confeitos. In: Manual Técnico do Seminário Produtos Diet e Light, Campinas, Junho de 2000, $154 \mathrm{p}$.

9. KALIL, S. J. Produção de inulinases por Kluyveromyces marxianus e purificação da enzima por cromatografia de troca iônica em coluna de leito expandido. Tese Doutorado, Universidade Estadual de Campinas, 1997,141 p.

10. LINKO, P. Lactose and lactitol. In: Nutritive sweeteners. London: Appied Science Publishers, 1982. cap. 6, p. 103-109.
11. LACTITOL. Disponível em < http://www.caloriecontrol.org/lactitol. html > Acesso em: 02 ago. 2006.

12. MARIE, S.; PIGGOTT, J. R. (Ed.). Handbook of sweeteners. Kluwer Academic Publishers, 1991, 320 p.

13. MEDEIROS, D. Diabetes tipo 2 aumenta entre adolescentes. Abril de 2004. Disponível em <http://www.cosmo.com.br/cidades/ campinas/2004/04/11/materia_cps_79289.shtm > Acesso em: 06 jan. 2006.

14. MeIlgaARD, D. M.; CIVILle, G. V.; CARR, B. T. Sensory Evaluation Techniques. Florida: CRC Press, 1999. 3. ed. $387 \mathrm{p}$.

15. MINIFIE, B. W. Chocolate, cocoa and confectionery: science and technology. 3. ed. New York: Chapman \& Hall, 1989. 118 p.

16. MOPPET, F. K. Polydextrose. In: Alternative Sweetners. Nova Iorque, Marcel Dekker, 1991, p. 401-421.

17. MOSKOWITZ, A. H. Maltitol and Hydrogenated Starch Hydrolysate. In: Alternative Sweetners. Nova Iorque, Marcel Dekker, 1991, p.259-282.

18. ORAFTI. Catálogo Técnico Raftiline, 2001.

19. PFIZER. Catálogo Técnico Litesse/Litesse III, 2000.

20. PROSKY, L. Cacao Bean and Its Products, AOAC INTERNATIONAL, 2000. Gaithersburg, Maryland - USA.

21. SPLENDA. Catálogo Sucralose. 2000.

22. SUCRALOSE. Disponível em: <http://www.caloriecontrol.org/ sucralos.html> Acesso em: 2 ago. 2006.

23. VIANNA, J. M. Saúde em movimento. Maio de 2002. Disponível em <http://www.saudeemmovimento.com.br/conteudos/ conteudo_exibe1.asp?cod_noticia $=626 . \mathrm{html}>$ Acesso em: 05 set. 2005.

24. VISSOTTO, F. Z. et al. Caracterização físico-química e reológica de chocolates comerciais tipo cobertura elaborados com gorduras alternativas. Brazilian Journal of Food Technology, v. 2, n. 1-2, p. 139-148, 1999.

25. VISSOTTO, F. Z.; GOMES, C. R.; BATISTA, B. J. L. Caracterização do comportamento reológico e da textura de chocolates sem sacarose. Brazilian Journal of Food Technology, v. 8, n. 2, p. 107-111, abr./jun. 2005

26. ZINK, R. Fabricação de balas duras, mastigávies e de goma dite. In: Apostila do Seminário de Produtos Diet e Light, Campinas, Junho de 2000, $154 \mathrm{p}$.

27. ZULETA, A., SAMBUCETTI, E. Inulin determination for food labeling. Agric. Food Chem., v. 49, n. 10, p. 4570-4572, 2001. 